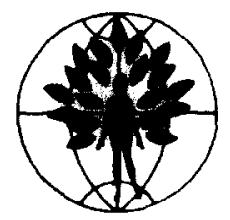

\title{
Motor vehicle crashes, pregnancy loss and preterm labor
}

\author{
M.D. Pearlman* \\ University of Michigan Medical Center, Department of Obstetrics and Gynecology, Ann Arbor, MI, USA
}

Received 2 August 1996; revised 23 October 1996; accepted 20 November 1996

\begin{abstract}
Trauma during pregnancy is remarkably common, and is greatly underestimated in terms of its contribution to both maternal and perinatal morbidity and mortality. Motor vehicle crashes, falls, assaults, including domestic violence, are all important mechanisms of injury, and clinical algorithms have been developed to manage the injured pregnant women. Focus on recognition and management of the most common injuries seen in pregnant women, namely abruptio placentae and uterine rupture are addressed through hemodynamic stabilization and continuous fetal and uterine contraction monitoring in the women injured beyond 24 weeks' gestation.
\end{abstract}

Keywords: Perinatal mortality; Trauma; Wounds and injuries; Pregnancy

\section{Introduction}

Worldwide, accidents and homicide are the leading cause of death among women of reproductive age [1]. This is also true in the United States (US), as indicated by data from the Center for Disease Control and Prevention [2]. These statistics are important in human economic terms,

\footnotetext{
${ }^{*}$ Tel.: +1 1313 7636670; fax: +1 3137647261 .
}

as the number of years of potential life loss is particularly devastating among these relatively young women.

In industrialized nations, approximately twothirds of all trauma during pregnancy results from motor vehicle crashes [3]. The remaining third is relatively evenly split between falls and assaults. In urban settings in the US, trauma is the leading cause of maternal mortality, with homicides and suicides responsible for a majority of these deaths $[4,5]$. 


\section{The mechanism of fetal and maternal injury in motor vehicle crashes}

In 1971, Crosby et al. reported a series of severe motor vehicle crashes in California involving pregnant women [6]. He concluded, based on this selected study population, that the major cause of fetal loss in severe motor vehicle crashes was the loss of maternal life. However, subsequent series reporting unselected motor vehicle crashes involving both severe and minor crashes clearly demonstrate that most fetal losses result from crashes involving minor maternal injuries [7-10]. The latter fact has great clinical import for two reasons: first, the vast (>90\%) preponderance of all maternal injuries are minor, and second, the evolving realization that pregnant women who suffer seemingly minor injuries, particularly minor blunt abdominal trauma, must be evaluated in a medical setting.

In most modern series of blunt abdominal trauma during pregnancy, more than $70 \%$ of fetal losses result from abruptio placentae [11] (Table 1). The mechanism of abruptio placentae resulting from trauma is based on the fundamental differences in tissue characteristics between the uterus and the placenta. The uterus consists of a significant proportion of elastic fibers whereas the placenta is largely devoid of elastic fibers. Thus, when an external deforming force is applied to the uterus, its inherent plasticity allows deformation. At the same time, the placenta cannot undergo similar deformation and a shearing effect is created at the uteroplacental interface. It is also thought that concomitant increases in amniotic fluid pressures propagate this shearing effect, further separating the placenta from the underlying decidua.
Because most blunt abdominal trauma occurs to the anterior uterine wall, one would expect that if the mechanism of separation were just displacement from the striking object, the risk of abruptio placentae would be greater when the placental location is anterior. However, the pathophysiologic mechanism of separation just described does not explain the finding that an anterior placental location does not appear to be a risk factor for abruptio placenta resulting from trauma [7]. Two alternative possibilities should be considered. Firstly, the contained mass within the amniotic fluid (i.e. the fetus) can either strike the placenta in any location and thus create a potential shear or, alternatively, pull the placenta by transmitting force via the umbilical cord. A second possibility that could explain the lack of importance of placental location on the likelihood of abruptio placentae is that traumatic deformation may set up a fluid wave within the uterus. If this were the case, a force striking the anterior uterine wall would cause elongation and narrowing of the uterus as the contained amniotic fluid is non-compressible. The fluid wave would then 'rebound' and expand horizontally causing a shortening and widening of the uterus. Again, because of the fundamental tissue differences between the uterus and placentae, a shearing effect of this interface could occur completely independent of placental location.

Regardless of whether or not abruptio placentae is present, traumatic injury to the uterus may also result in destabilization of lysosomal enzymes which can initiate prostaglandin production [3]. This is the proposed mechanism presumed to cause preterm labor associated with trauma. At the present time, the true incidence of preterm labor following trauma during pregnancy is not

Table 1

Fetal loss following blunt abdominal trauma (combined data from 6 studies $^{\mathrm{a}}$ )

\begin{tabular}{lcccc}
\hline Trauma severity & $n(\%)$ & Abruptio placentae (\%) & Direct fetal injury (\%) & Fetal loss (\%) \\
\hline Life threatening $^{\mathrm{b}}$ & $32(6.2)$ & $12(37.5)$ & $1(3.1)$ & $13(40.6)$ \\
Non-life threatening $^{\mathrm{a}}$ & $487(93.8)$ & $8(1.6)$ & $4(0.8)$ & $8(1.6)$ \\
\hline
\end{tabular}

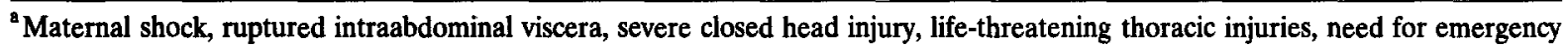
laparotomy for maternal indications. Maternal mortality excluded.

${ }^{\mathrm{b}}$ Studies available on request. 


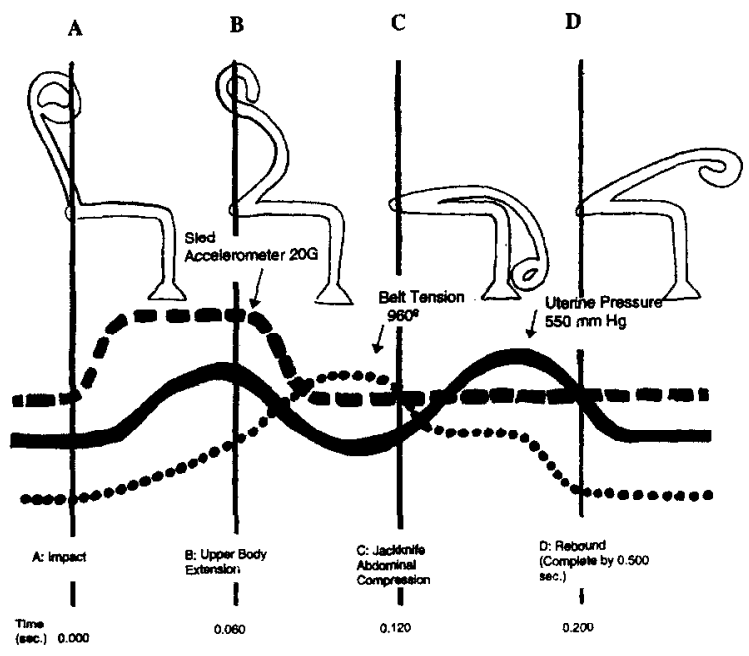

Fig. 1. Impact sequence. (Reproduced with permission from Crosby, W.M. et al., Am. J. Obstet. Gynecol. 101 (1968) 110.) known with certainty, but appears to be under $5 \%$ in two reported studies $[7,9]$.

\subsection{Animal experiments}

In a series of experimental crashes involving pregnant baboons, the effect of force transmission to the uterine compartment was evaluated (see Fig. 1) $[12,13]$. In these experiments, pregnant baboons were placed in two point restraint in a Hyge sled accelerometer which simulates an automobile crash under conditions of rapid deceleration. At $25 \mathrm{mph}$, a biphasic increase in intrauterine pressure is experienced. The first increase results from sudden deceleration of the

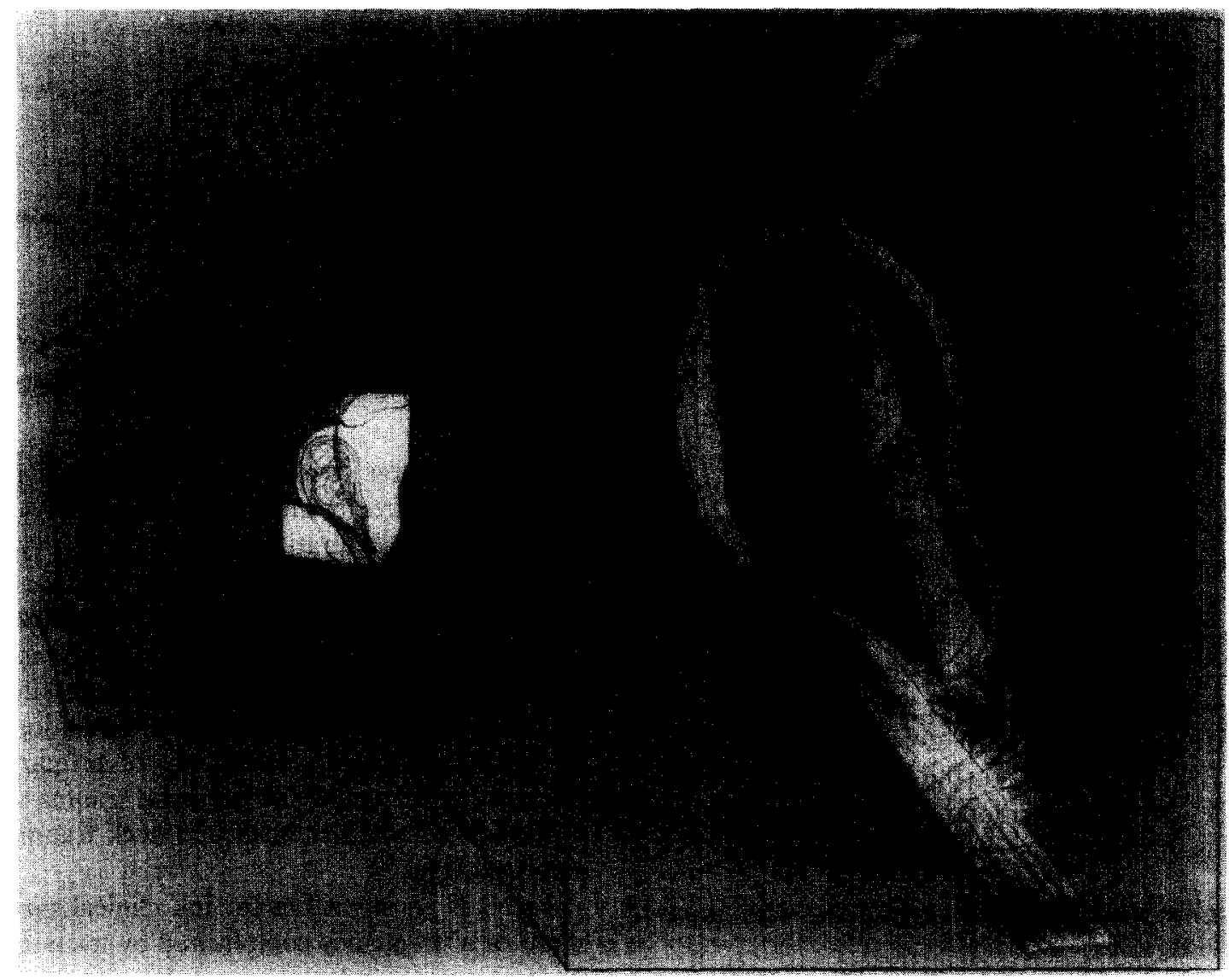

Fig. 2. Proper seat belt placement. (Copyright University of Michigan.) 
pelvis, stopped by the lap belt. At the same time, the uterus continues to move forward striking the anterior abdominal wall. This results in an increase in intrauterine pressure approaching 500 $\mathrm{mmHg}$. Later during this crash sequence, the upper torso of the animal is thrown forward essentially collapsing around the pregnant uterus creating a second increase in intrauterine pressure, approaching $550 \mathrm{mmHg}$. From this and other experiments with animals held by three point restraints (lap belt and shoulder belt), it is clear that the second increase in intrauterine pressure can be climinated by preventing the torso from collapsing around the pregnant uterus. This results in a decrease in fetal mortality from $100 \%$ to $40 \%$ [13].

\subsection{Human studies}

Unfortunately, very little data exists on the effect of modern seat belt and restraint usage on the loss of human fetal life. The data from early series demonstrated only that the use of two point restraints lowered the likelihood of loss of maternal life [6]. Virtually no information exists on the currently required three point restraint system, or with airbag use. A unique new study is underway using a pregnant anthropomorphic test device (crash dummy) at the University of Michigan and the General Motors Corporation [14]. This device may help clarify the mechanism of pregnancy loss during automobile crashes, and provide insight into the reduction of the physical forces which lead to pregnancy loss. Currently, it is recommended that all pregnant women utilize both a lap belt and shoulder harness while occupants in automobiles [15]. The lap belt should be placed as low as possible, beneath the bulge of the uterus, and the shoulder belt should lie to the side of the uterus, run between the breasts and over the mid portion of the clavicle (Fig. 2). No specific recommendations have been made regarding air bag use as it relates to pregnancy.

\section{Clinical aspects of treating patients with motor vehicle injuries}

Because the uterine vasculature is maximally dilated in the resting physiologic state, any event

\section{STABILIZATION}
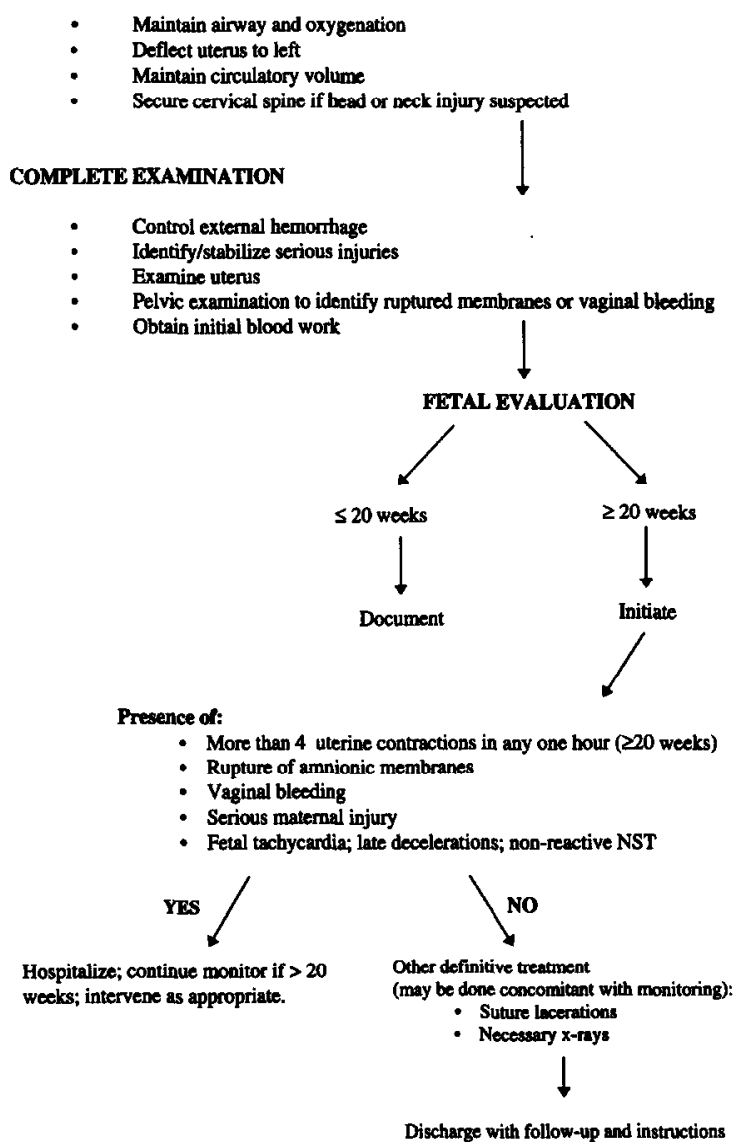

Fig. 3. Algorithm for management of trauma during pregnancy.

which interferes with maternal hemodynamic stability can directly interfere with fetal oxygenation. Similarly, alterations in maternal oxygenation can profoundly effect fetal oxygenation. Under these circumstances, maternal stabilization has top priority in the management of the injured pregnant woman [3]. Securing and maintaining a patent airway, assuring adequate ventilation and adequate circulating volume are of primary importance in managing the pregnant trauma victim regardless of whether head and neck trauma has occurred (Fig. 3).

Fig. 3 is an algorithm for the clinical management of a pregnancy complicated by trauma. The early diagnosis and management of abruptio placentae is a major concern. This is true not only because it is the leading cause of fetal loss fol- 
lowing trauma, but also that timely intervention can substantially improve outcome of the fetus [7]. Two other less common causes of fetal loss following trauma are fetal-maternal hemorrhage and direct fetal injury [7-9,17]. Unfortunately, the ability of subsequent clinical management to affect outcome may be considerably less in both latter circumstances.

Existing policies and algorithms have understandably concentrated on an ability to establish an early diagnosis of abruptio placentae $[7-9,16]$. Central to these management guidelines has been the concomitant use of continuous electronic fetal and uterine contraction monitoring in the latter half of pregnancy. In three separate studies, the presence of frequent uterine activity has been the most sensitive predictor of abruptio placentae [7-9]. In one of these, all patients who developed abruptio placentae had at least 8 uterine contractions per hour during an initial 4-h monitoring period [7]. The exact duration that monitoring must be continued is unknown, protocols with monitoring periods ranging from $2-24 \mathrm{~h}$ have been successfully utilized in the clinical management following trauma during pregnancy [16].

Fetal-maternal hemorrhage occurs approximately 4 times more frequently following trauma than it does in uninjured controls $[7,9,17]$ of comparable gestational age. Nonetheless, fetal loss resulting from exsanguination into the maternal circulation is rare, and the clinical utility of using the Kleihauer-Betke assay as a tool to diagnose significant fetal-maternal hemorrhage has not been proven. Typically, the most prompt initial management of significant fetal maternal hemorrhage will be through evaluation of the fetal heart tracing. Fetal heart rate abnormalities due to acute FMH include tachycardia, bradycardia, or late decelerations in a sinusoidal pattern. Complications due to FMH are much more likely to be detected through fetal heart rate abnormalities than through Kleihauer-Betke testing, and routine use of Kleihauer-Betke testing is not rapid enough nor sufficiently sensitive to detect fetal abnormalities. Nonetheless, it is likely there exists a risk of isoimmunization in the $\mathrm{Rh}$ negative woman, and $\mathrm{Rh}$ immune globulin should be administered to all $\mathrm{Rh}$ negative women following trauma during pregnancy. For this, the Kleihauer-Betke assay is more useful in determining the $\mathrm{Rh}$ immune globulin dose necessary in those rare patients with massive fetal-maternal hemorrhage than it is when used as a routine screen for clinically significant FMH; (300 $\mu \mathrm{g} \mathrm{Rh}$ immune globulin protects against a $30 \mathrm{cc} \mathrm{FMH}$ ).

The experience gained from large series supports the protocol shown in Fig. 3. All women beyond 20-24 weeks who suffer blunt trauma should be monitored for a minimum of $4 \mathrm{~h}$. The finding of frequent uterine activity, non-reassuring fetal heart toncs, serious maternal injurics which would otherwise require hospitalization, rupture of membranes, vaginal bleeding, or significant uterine tenderness, would dictate prolonged observation with continuous monitoring and/or intervention depending on the clinical circumstance. Obviously, gestational age at the time of injury will play an important role in determining whether intervention (immediate delivery) is appropriate.

\section{Comments}

The importance of accidents on loss of fetal life is virtually unknown whereas accidents and homicides are well recognized as a cause of loss of life among women. Approximately 4 million births occur each year in the United States. The incidence of trauma during pregnancy is estimated to vary between $6 \%$ and $7 \%$ [7-9]. In these series, minor and/or non-life threatening injuries during pregnancy resulted in a $1-5 \%$ pregnancy loss, in contrast to life threatening traumatic events which resulted in a $40-50 \%$ fetal loss rate. Using these data, one can extrapolate that between 2600 and 13000 pregnancies are lost each year in the United States as a result of trauma. The economic impact of the loss of each unborn child to its family or to society is unclear. However, assuming that each of these fetuses lived to age 72 , the potential years of life lost are between 172800 and 936000.

Only coordinated and effective effort by medical personnel can reduce the mortality rate after motor vehicle trauma. Other reductions must come through prevention. Attention to issues such 
as passenger restraint systems for pregnant women can substantially reduce both maternal and fetal morbidity and mortality.

\section{References}

[1] World Health Organization. World Health Statistics. Geneva: WHO. 1992.

[2] Kochanek KD, Hudson BL. Centers for Disease Control and Prevention, Monthly Vital Statistics Report: Advance Report of Final Mortality Statistics - 1992, 1994; 43: 1-13.

[3] Pearlman MD, Tintinalli JE, Lorenz RP. Blunt trauma during pregnancy. N Engl J Med 1990; 323: 1609.

[4] Fildes J, Reed L, Jones N, Martin M, Barrett J. Trauma: the leading cause of maternal death. J Trauma 1992; 32: 643-645.

[5] Dannenberg AL, Carter DM, Lawson HW, Ashton DM, Dorfman SF, Graham EH. Homicide and other injuries as causes of maternal death in New York city, 1987 through 1991. Am J Obstet Gynecol 1995; 172: 1557-1564.

[6] Crosby WM, Costiloe JP. Safety of lap-belt restraint for pregnant victims of automobile collisions. $\mathrm{N}$ Engl J Med 1971; 284: 632.

[7] Pearlman MD, Tintinalli JE, Lorenz RP. A prospective controlled study of outcome after trauma during pregnancy. Am J Obstet Gynecol 1990; 162: 1502.

[8] Williams JK, McClain L, Rosemurgy AS, Colorado NM.
Evaluation of blunt abdominal trauma in the third trimester of pregnancy: maternal and fetal considerations. Obstet Gynecol 1990; 75: 33.

[9] Goodwin TM, Breen MT. Pregnancy outcome and fetomaternal hemorrhage after noncatastrophic trauma. Am J Obstet Gynecol 1990; 162: 665.

[10] Dahmus MA, Sibai BM. Blunt abdominal trauma: are there any predictive factors for abruptio placentae or maternal-fetal distress. Am J Obstet Gynecol 1993: 169: 1054.

[11] Pearlman MD, Tintinalli JE. Evaluation and treatment of the gravida and fetus following trauma during pregnancy. Obstet Gynecol Clin N Am 1991; 18: 371-381.

[12] Crosby WM, Snyder RG, Snow CC, Hanson PG. Impact injuries in pregnancy. I. Experimental studies. Am J Obstet Gynecol 1968; 101: 110.

[13] Crosby WM, King AI, Stout CC. Fetal survival following impact: improvement with shoulder harness restraint. Am J Obstet Gynecol 1972; 112: 1101.

[14] Pearlman MD, Viano D. Auto crash simulation using the first pregnant crash test dummy. Am J Obstet Gynecol 1996; 175: 977-981.

[15] ACOG Technical Bulletin. Automobile passenger restraints for children and pregnant women. Number 151, January 1991.

[16] ACOG Technical Bulletin. Trauma during pregnancy. Number 161, November 1991.

[17] Rose PG, Strohm PL, Zuspan FR. Fetomaternal hemorrhage following trauma. Am J Obstet Gynecol 1985; 153: 844 . 